\title{
Demonstration of optical beating in the reflectance and transmittance spectra of multilayer structures
}

\author{
${ }^{1}$ Kosobutskyy P.S., ${ }^{2}$ Bilyj J.M. and ${ }^{2}$ Kushnir O.P. \\ ${ }^{1}$ Lviv Polytechnic National University, P. O. Box 4544, 79053 Lviv, Ukraine, \\ e-mail: petkosob@yahoo.com \\ ${ }^{2}$ Lviv National Agrarian University, 1 V. Velykyi St., Dublyany, \\ 80381 Lviv Region, Ukraine, E-mail: o-p-ku@ukr.net
}

Received: 25.10 .2010

After revision: 24.03.2011

\begin{abstract}
A analogy between optical beating in the inverse transmittance spectrum of transparent double-layer structure and a beating phenomenon known from the standard vibration theory has been considered. We have determined analytical conditions under which the optical beating manifests itself the most clearly in the interference spectra of multilayer structures.
\end{abstract}

Keywords: multilayer structure, spectral envelopes, optical beating.

PACS: $63.22 .+\mathrm{m} ;$ 78.30-j; 78.30.Fs; 78.66.Hf

UDC: $535.417,535.016$.

\section{Introduction}

It is well known that a plane-parallel Fabry-Perot resonator represents a multifunctional device with very wide application range, including filtering of signals [1], optical communications [2, 3], sensors [4, 5], etc. Usually, it is analysed basing on coherent summation of signals, with consequent calculations of interference and polarisation patterns. This represents a basis of interference methods for parameters metering [6], along with strong complement of multibeam interference envelope technique [7, 8]. A number of analytical relations for the envelopes of both the minimums and maximums of the interference spectra for the structures consisting of two and three layers have been derived in the work [9] using computer modelling of amplitude-and-phase Fabry-Perot spectra. These relations correspond to different possible ratios between the phase thicknesses of the layers. However, the studies $[9,10]$ do not contain physical explanations of the phenomenon. In the present article we derive expressions for the envelopes in frame of analysis for the regularities of optical beating, which is demonstrated for the interference spectra of the structures with two (or more) layers. Further on we will use a term 'inverse transmittance spectrum' recently used in the literature, in order to make a relevant analogy. It is stipulated by simplicity and efficiency of analytical expressions for the inverse transmittance, $1 / T$, when analysing multilayer interference coatings [11]. 


\section{Optical beating in the reflectance, transmittance and inverse transmittance spectra of multilayer structures}

Unlike single-layered structures, the envelopes of the reflectance $(R(\lambda))$, transmittance $(T(\lambda))$ and inverse transmittance $(W(\lambda)=1 / T(\lambda))$ spectra of multilayer structures are oscillating, and the peak-to-peak amplitude of these oscillations depends on the light wavelength:

$$
\begin{aligned}
& \Delta R(\lambda)=R^{\max }(\lambda)-R^{\min }(\lambda), \\
& \Delta T(\lambda)=T^{\max }(\lambda)-T^{\min }(\lambda), \\
& \Delta W(\lambda)=W^{\max }(\lambda)-W^{\min }(\lambda),
\end{aligned}
$$

where $R^{\max }(\lambda), T^{\max }(\lambda), W^{\max }(\lambda)$ and $R^{\min }(\lambda), T^{\min }(\lambda), W^{\min }(\lambda)$ are respectively the envelopes of maximums and minimums in these spectra. The less the ratios $\frac{\Delta R^{\min }}{\Delta R^{\max }}$ (for the reflectance spectrum), $\frac{\Delta T^{\min }}{\Delta T^{\max }}$ (for the transmittance spectrum) and $\frac{\Delta W^{\min }}{\Delta W^{\max }}$ (for the inverse transmittance spectrum), the more clearly the optical beating manifests itself in the spectra in the shape of pulsations $\Delta R(\lambda), \Delta T(\lambda)$ or $\Delta W(\lambda)$. Here $\Delta R^{\min , \max }$, $\Delta T^{\min , \max }$ and $\Delta W^{\min , \max }$ are the minimum and maximum values of the peak-to-peak amplitudes (see Fig. 1) in the appropriate spectra observed inside the wavelength interval under examination.

\subsection{Optical beating in the inverse transmittance spectrum for a transparent double-layer structure as an analogue of beating in the vibration theory}

One can draw a mathematical analogy between optical beatings observed in the inverse transmittance spectrum $W(\lambda)$ of a transparent double-layer structure and a phenomenon of beating known from the standard vibration theory. It is well-known that the latter arises when two harmonic oscillations with equal amplitudes and close frequencies are superposed $[12,13]$. For this purpose, we take into consideration the expression for the amplitude transmittance $\tilde{t}_{0,3}=\frac{t_{0,1} t_{1,2} t_{2,3} e^{-i \delta_{1}} e^{-i \delta_{2}}}{1+r_{1,2} r_{2,3} e^{-2 i \delta_{2}}+r_{0,1} r_{1,2} e^{-2 i \delta_{1}}+r_{0,1} r_{2,3} e^{-2 i \delta_{1}} e^{-2 i \delta_{2}}} \quad$ [14] and write down the inverse transmittance $W=\frac{n_{0}}{n_{3}} \frac{\cos \beta_{0}}{\cos \beta_{3}} \frac{1}{\tilde{t}_{0,3}} \frac{1}{\tilde{t}_{0,3}^{*}}$ in the following form:

$$
W=A+B\left(S_{1} \cos \left(2 \delta_{1}\right)+S_{2} \cos \left(2 \delta_{2}\right)+S_{3} \cos \left(2 \delta_{1}+2 \delta_{2}\right)+S_{4} \cos \left(2 \delta_{1}-2 \delta_{2}\right)\right) \text {, }
$$

where $\quad A=\frac{n_{0}}{n_{3}} \frac{\cos \beta_{0}}{\cos \beta_{3}} \frac{1}{t_{0,1}^{2} t_{1,2}^{2} t_{2,3}^{2}}\left(1+r_{1,2}^{2} r_{2,3}^{2}+r_{0,1}^{2} r_{1,2}^{2}+r_{0,1}^{2} r_{2,3}^{2}\right), \quad B=\frac{n_{0}}{n_{3}} \frac{\cos \beta_{0}}{\cos \beta_{3}} \frac{1}{t_{0,1}^{2} t_{1,2}^{2} t_{2,3}^{2}}$, $S_{1}=2 r_{0,1} r_{1,2}+2 r_{0,1} r_{1,2} r_{2,3}^{2}, \quad S_{2}=2 r_{1,2} r_{2,3}+2 r_{0,1}^{2} r_{1,2} r_{2,3}, \quad S_{3}=2 r_{0,1} r_{2,3}$, and $S_{4}=2 r_{0,1} r_{1,2}^{2} r_{2,3}$. 
Here $r_{i, i+1}$ and $t_{i, i+1}(i=0,1,2)$ denote respectively the Fresnel reflection and transmission coefficients for single interfaces $i-(i+1)[14], \delta_{j}=\frac{2 \pi d_{j} n_{j} \cos \beta_{j}}{\lambda}(j=1,2)$ the phase thickness, $n_{j}$ the refractive index, $d_{j}$ the thickness of layer $j$, and $\beta_{j}$ the angle of light propagation in this layer.

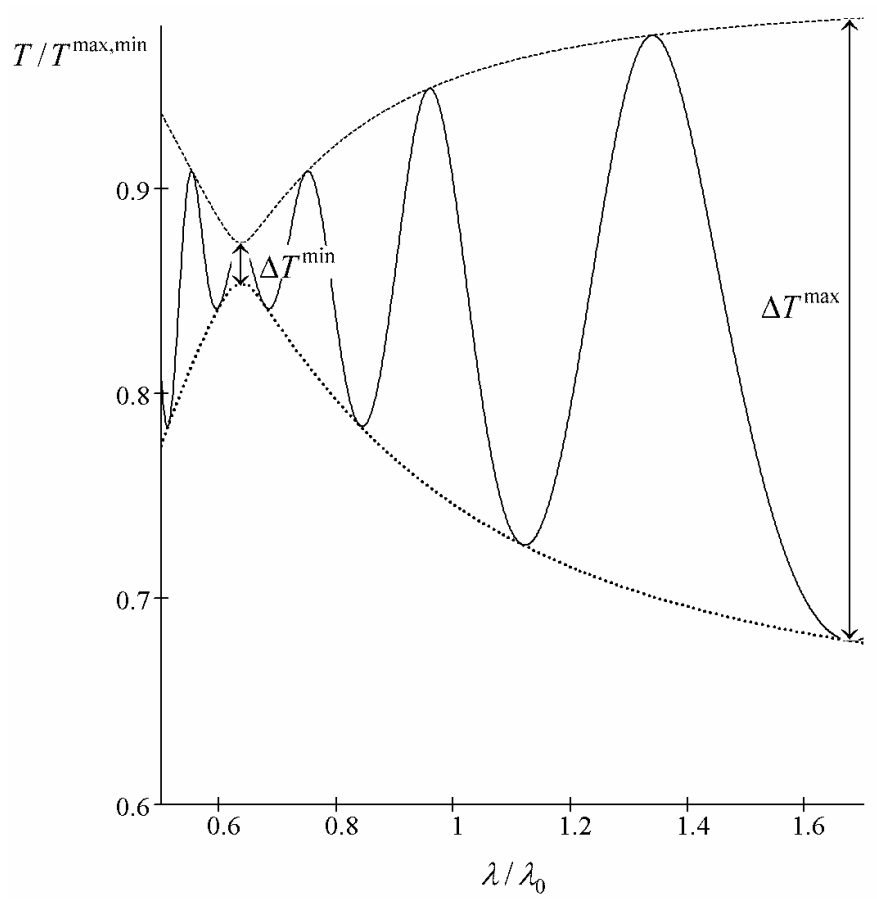

Fig. 1. Transmittance at normal incidence ( $T$, solid curve) and envelopes of its minimums ( $T^{\mathrm{min}}$, dotted curve) and maximums ( $T^{\max }$, dashed curve) for a transparent double-layer structure with the refraction indices $n_{0}=1$, $n_{1}=1.38, \quad n_{2}=1.85, \quad n_{3}=4.0$ and the phase thicknesses of layers $\delta_{1}=1 \mathrm{rad}$ and $\delta_{2}=10 \mathrm{rad}$ at $\lambda=\lambda_{0}$.

Whenever $\frac{\Delta W^{\min }}{\Delta W^{\max }}=0$, the optical beating in the inverse transmittance spectrum of double-layer structures is seen very clearly in the four following cases:

$$
d_{1} n_{1} \cos \beta_{1} \gg d_{2} n_{2} \cos \beta_{2}, r_{1,2}=r_{2,3},
$$

$$
\begin{aligned}
W= & A+2 r_{0,1} r_{1,2} B\left(\left(1+r_{1,2}^{2}\right) \cos \left(2 \delta_{1}\right)+r_{0,1} r_{1,2} \cos \left(2 \delta_{2}\right)+\cos \left(2 \delta_{1}+2 \delta_{2}\right)+r_{1,2}^{2} \cos \left(2 \delta_{1}-2 \delta_{2}\right)\right) \\
& +2 r_{1,2}^{2} B \cos \left(2 \delta_{2}\right) ;
\end{aligned}
$$

$$
d_{1} n_{1} \cos \beta_{1} \gg d_{2} n_{2} \cos \beta_{2}, r_{1,2}=-r_{2,3},
$$




$$
\begin{aligned}
W= & A+2 r_{0,1} r_{1,2} B\left(\left(1+r_{1,2}^{2}\right) \cos \left(2 \delta_{1}\right)-r_{0,1} r_{1,2} \cos \left(2 \delta_{2}\right)-\cos \left(2 \delta_{1}+2 \delta_{2}\right)-r_{1,2}^{2} \cos \left(2 \delta_{1}-2 \delta_{2}\right)\right) \\
& -2 r_{1,2}^{2} B \cos \left(2 \delta_{2}\right) ; \\
& \quad(3) \quad d_{1} n_{1} \cos \beta_{1} \ll d_{2} n_{2} \cos \beta_{2}, r_{0,1}=r_{1,2}, \\
W= & A+2 r_{1,2} r_{2,3} B\left(r_{1,2} r_{2,3} \cos \left(2 \delta_{1}\right)+\left(1+r_{1,2}^{2}\right) \cos \left(2 \delta_{2}\right)+\cos \left(2 \delta_{1}+2 \delta_{2}\right)+r_{1,2}^{2} \cos \left(2 \delta_{1}-2 \delta_{2}\right)\right) \\
& +2 r_{1,2}^{2} B \cos \left(2 \delta_{1}\right) ; \\
& (4) \quad d_{1} n_{1} \cos \beta_{1} \ll d_{2} n_{2} \cos \beta_{2}, r_{0,1}=-r_{1,2}, \\
W= & A+2 r_{1,2} r_{2,3} B\left(-r_{1,2} r_{2,3} \cos \left(2 \delta_{1}\right)+\left(1+r_{1,2}^{2}\right) \cos \left(2 \delta_{2}\right)-\cos \left(2 \delta_{1}+2 \delta_{2}\right)-r_{1,2}^{2} \cos \left(2 \delta_{1}-2 \delta_{2}\right)\right) \\
- & -2 r_{1,2}^{2} B \cos \left(2 \delta_{1}\right) .
\end{aligned}
$$

While drawing the analogy, we will also consider a variant of the structure, for which the refractive indices of the neighbouring layers differ insignificantly from each other $\left(\left|n_{i+1}-n_{i}\right|<0.5\right)$. Then we may ignore small summands in Eqs. (6), (8), (10) and (12), assuming that the conditions $r_{1,2}^{2} \ll 1,\left|r_{0,1} r_{1,2}\right| \ll 1$ and $\left|r_{1,2} r_{2,3}\right| \ll 1$ are valid.

If the structure parameters satisfy the conditions described by Eqs. (5), then the inverse transmittance (see Fig. 2) is given by

$$
\begin{aligned}
& W=A+B\left(2 r_{0,1} r_{1,2} \cos \left(2 \delta_{1}+\delta_{2}-\delta_{2}\right)+2 r_{0,1} r_{1,2} \cos \left(2 \delta_{1}+\delta_{2}+\delta_{2}\right)+2 r_{1,2}^{2} \cos \left(2 \delta_{2}\right)\right) \\
& =C_{2}^{+}+4 r_{0,1} r_{1,2} B \cos \left(2 \pi d_{2} n_{2} \cos \beta_{2} \frac{1}{\lambda}\right) \cos \left(2 \pi\left[2 d_{1} n_{1} \cos \beta_{1}+d_{2} n_{2} \cos \beta_{2}\right] \frac{1}{\lambda}\right),
\end{aligned}
$$

where $C_{2}^{+}=A+2 r_{1,2}^{2} B \cos \left(4 \pi d_{2} n_{2} \cos \beta_{2} \frac{1}{\lambda}\right)$. In the case given by Eqs. (7) the inverse transmittance may be represented as

$$
\begin{aligned}
& W=A+B\left(2 r_{0,1} r_{1,2} \cos \left(2 \delta_{1}+\delta_{2}-\delta_{2}\right)-2 r_{0,1} r_{1,2} \cos \left(2 \delta_{1}+\delta_{2}+\delta_{2}\right)-2 r_{1,2}^{2} \cos \left(2 \delta_{2}\right)\right) \\
& =C_{2}^{-}+4 r_{0,1} r_{1,2} B \sin \left(2 \pi d_{2} n_{2} \cos \beta_{2} \frac{1}{\lambda}\right) \sin \left(2 \pi\left[2 d_{1} n_{1} \cos \beta_{1}+d_{2} n_{2} \cos \beta_{2}\right] \frac{1}{\lambda}\right),
\end{aligned}
$$

where $C_{2}^{-}=A-2 r_{1,2}^{2} B \cos \left(4 \pi d_{2} n_{2} \cos \beta_{2} \frac{1}{\lambda}\right)$. In the case (9) we have

$$
\begin{aligned}
& W=A+B\left(2 r_{1,2} r_{2,3} \cos \left(2 \delta_{2}+\delta_{1}-\delta_{1}\right)+2 r_{1,2} r_{2,3} \cos \left(2 \delta_{2}+\delta_{1}+\delta_{1}\right)+2 r_{1,2}^{2} \cos \left(2 \delta_{1}\right)\right) \\
& =C_{1}^{+}+4 r_{1,2} r_{2,3} B \cos \left(2 \pi d_{1} n_{1} \cos \beta_{1} \frac{1}{\lambda}\right) \cos \left(2 \pi\left[2 d_{2} n_{2} \cos \beta_{2}+d_{1} n_{1} \cos \beta_{1}\right] \frac{1}{\lambda}\right),
\end{aligned}
$$

where $C_{1}^{+}=A+2 r_{1,2}^{2} B \cos \left(4 \pi d_{1} n_{1} \cos \beta_{1} \frac{1}{\lambda}\right)$. Finally, if the parameters of the structure satisfy the conditions given by Eq. (11), one gets 


$$
\begin{aligned}
& W=A+B\left(2 r_{1,2} r_{2,3} \cos \left(2 \delta_{2}+\delta_{1}-\delta_{1}\right)-2 r_{1,2} r_{2,3} \cos \left(2 \delta_{2}+\delta_{1}+\delta_{1}\right)-2 r_{1,2}^{2} \cos \left(2 \delta_{1}\right)\right) \\
& =C_{1}^{-}+4 r_{1,2} r_{2,3} B \sin \left(2 \pi d_{1} n_{1} \cos \beta_{1} \frac{1}{\lambda}\right) \sin \left(2 \pi\left[2 d_{2} n_{2} \cos \beta_{2}+d_{1} n_{1} \cos \beta_{1}\right] \frac{1}{\lambda}\right)
\end{aligned}
$$

where $C_{1}^{-}=A-2 r_{1,2}^{2} B \cos \left(4 \pi d_{1} n_{1} \cos \beta_{1} \frac{1}{\lambda}\right)$.

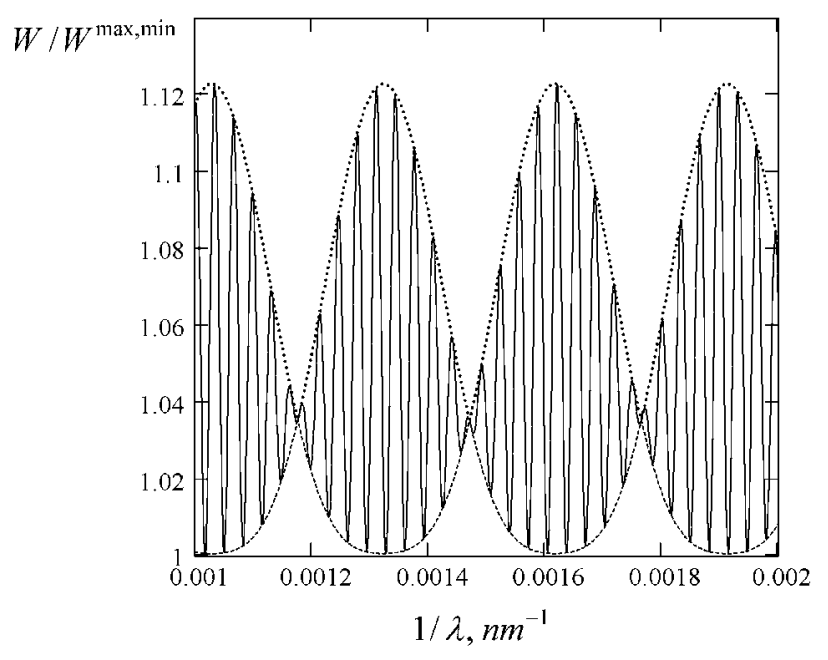

Fig. 2. Inverse transmittance at normal incidence ( $W$, solid curve) and envelopes of its minimums ( $W^{\mathrm{min}}$, dashed curve) and maximums ( $W^{\text {max }}$, dotted curve) for a transparent double-layer structure with the parameters $n_{0}=1$, $n_{1}=1.45, \quad n_{2}=1.70, \quad n_{3}=1.99$, $d_{1}=10 \mu \mathrm{m}, d_{2}=1 \mu \mathrm{m}$ (the conditions of Eqs. (5) is satisfied).

It is known that a beating appears when two harmonic oscillations (see Fig. 3) with equal amplitudes $a$ and close frequencies $w$ and $w+\Delta w(\Delta w \ll w)$ are summing up [12,13]:

$$
\begin{aligned}
& y=a \cos (w t)+a \cos ((w+\Delta w) t)=a \cos \left(\left(w+\frac{\Delta w}{2}-\frac{\Delta w}{2}\right) t\right)+a \cos \left(\left(w+\frac{\Delta w}{2}+\frac{\Delta w}{2}\right) t\right) \\
& =2 a \cos \left(\frac{\Delta w}{2} t\right) \cos \left(\left(w+\frac{\Delta w}{2}\right) t\right) .
\end{aligned}
$$

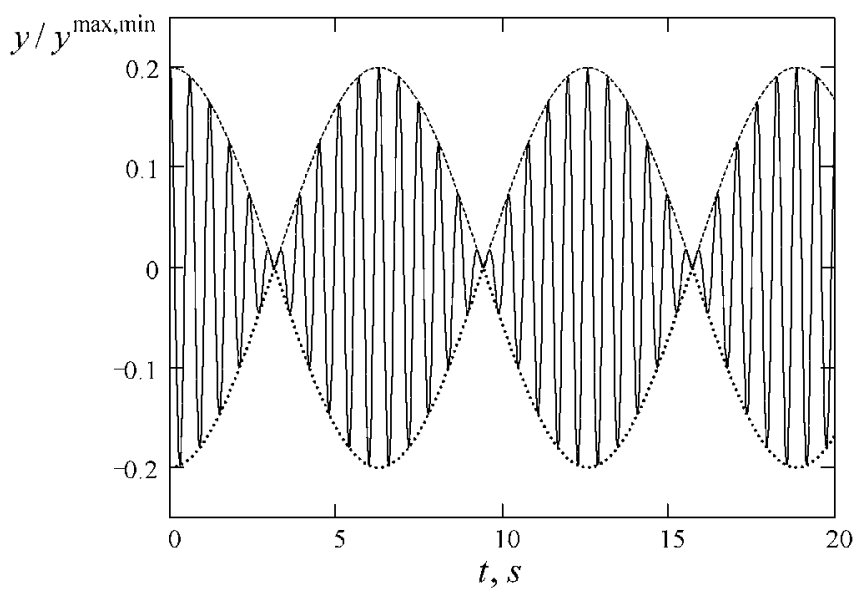

Fig. 3. Illustration of beating arising as a result of summation of two vibrations given by Eq. (17) with the parameters $a=0.1, \quad w=10 \mathrm{rad} / \mathrm{s} \quad$ and $\Delta w=1 \mathrm{rad} / \mathrm{s}$ : solid curve corresponds to superposition of two harmonic oscillations $y$, dotted curve to envelope of minimums $y^{\min }$, and dashed curve to envelope of maximums $y^{\max }$.

Let us compare Eqs. (13) and (17) for the case (5), Eqs. (14) and (17) for the case (7), Eqs. (15) and (17) for the case (9), and Eqs. (16) and (17) for the case (11). This draws a conclusion that the optical beatings are available in the inverse transmittance 
spectra observed for the double-layer structures of the types considered above. They represent a clear analogue of the beating known from the vibration theory (see Eq. (17)). Unambiguous analogies (Table 1) can be made among the parameters of the inverse transmittance spectrum, for which the optical beating takes place, and the parameters of the mentioned vibration process. Modulation curves of the inverse transmittance (see Table 1) taken inside certain inverse-wavelength intervals are envelopes of the maximums $W^{\text {max }}$, while inside the other intervals they represent envelopes of the minimums $W^{\text {min }}$.

Table 1. Analogy of the optical beating and the beating known from the vibration theory (see Eq. (17)).

\begin{tabular}{|c|c|}
\hline $\begin{array}{c}\text { Inverse transmittance spectrum of transparent } \\
\text { double-layer structure (Eq. (4)) }\end{array}$ & $\begin{array}{l}\text { Sum of two harmonic vibrations } \\
\text { (Eq. (17)) }\end{array}$ \\
\hline $\begin{array}{c}\text { Inverse wavelength } \\
1 / \lambda\end{array}$ & Time $t$ \\
\hline $\begin{array}{l}4 \pi d_{1} n_{1} \cos \beta_{1} \text { for the cases (5) and (7); } \\
4 \pi d_{2} n_{2} \cos \beta_{2} \text { for the cases (9) and (11) }\end{array}$ & $\begin{array}{c}\text { Frequency } w \text { of one of the vibra- } \\
\text { tions }\end{array}$ \\
\hline $4 \pi\left(d_{1} n_{1} \cos \beta_{1}+d_{2} n_{2} \cos \beta_{2}\right)$ & $\begin{array}{c}\text { Frequency } w+\Delta w \text { of the other } \\
\text { vibration }\end{array}$ \\
\hline $\begin{array}{l}\text { Condition for the beating under which peak-to-peak } \\
\text { amplitude of oscillations reaches a value close to zero: } \\
d_{2} n_{2} \cos \beta_{2} \ll d_{1} n_{1} \cos \beta_{1} \text { for the cases (5) and (7); } \\
d_{1} n_{1} \cos \beta_{1} \ll d_{2} n_{2} \cos \beta_{2} \text { for the cases (9) and (11) }\end{array}$ & $\Delta w \ll w$ \\
\hline $\begin{array}{l}C_{2}^{+}+4 r_{0,1} r_{1,2} B \cos \left(2 \pi d_{2} n_{2} \cos \beta_{2} \frac{1}{\lambda}\right) \text { for the case (5); } \\
C_{2}^{-}+4 r_{0,1} r_{1,2} B \sin \left(2 \pi d_{2} n_{2} \cos \beta_{2} \frac{1}{\lambda}\right) \text { for the case (7); } \\
C_{1}^{+}+4 r_{1,2} r_{2,3} B \cos \left(2 \pi d_{1} n_{1} \cos \beta_{1} \frac{1}{\lambda}\right) \text { for the case (9); } \\
C_{1}^{-}+4 r_{1,2} r_{2,3} B \sin \left(2 \pi d_{1} n_{1} \cos \beta_{1} \frac{1}{\lambda}\right) \text { for the case (11) }\end{array}$ & Modulated curve $2 a \cos \left(\frac{\Delta w}{2} t\right)$ \\
\hline
\end{tabular}

When dealing with the function $y(t)$ given by Eq. (17), it would also be convenient to introduce the envelopes for the maximums $\left(y^{\max }\right)$ and the minimums $\left(y^{\min }\right)$ :

$$
\begin{aligned}
& y^{\max }=\left|2 a \cos \left(\frac{\Delta w}{2} t\right)\right|, \\
& y^{\min }=-\left|2 a \cos \left(\frac{\Delta w}{2} t\right)\right|,
\end{aligned}
$$

thus drawing the analogy with the envelopes of the inverse transmittance spectrum even more vivid. That these functions are really the envelopes is evidenced by fulfilment of the following inequalities: 


$$
\begin{gathered}
y^{\max }-y=\left|2 a \cos \left(\frac{\Delta w}{2} t\right)\right|-2 a \cos \left(\frac{\Delta w}{2} t\right) \cos (w t) \\
=\left|2 a \cos \left(\frac{\Delta w}{2} t\right)\right|\left(1-\cos (w t) \operatorname{sgn}\left[\cos \left(\frac{\Delta w}{2} t\right)\right] \mid \geq 0\right. \\
y-y^{\min }=2 a \cos \left(\frac{\Delta w}{2} t\right) \cos (w t)+\left|2 a \cos \left(\frac{\Delta w}{2} t\right)\right|, \\
=\left|2 a \cos \left(\frac{\Delta w}{2} t\right)\right|\left(\cos (w t) \operatorname{sgn}\left[\cos \left(\frac{\Delta w}{2} t\right)\right]+1\right) \geq 0 \\
\text { where } \operatorname{sgn}\left[\cos \left(\frac{\Delta w}{2} t\right)\right]=\left\{\begin{array}{l}
\left.1, \text { when } \cos \left(\frac{\Delta w}{2} t\right) \geq 0\right] \\
-1, \text { when } \cos \left(\frac{\Delta w}{2} t\right)<0
\end{array}\right\} .
\end{gathered}
$$

The analogues of the envelopes $y^{\max , \min }$ found in the inverse transmittance spectrum are the parameters

$$
W^{\max }=\left\{\begin{array}{l}
C_{2}^{+}+\left|4 r_{0,1} r_{1,2} B \cos \left(2 \pi d_{2} n_{2} \cos \beta_{2} \frac{1}{\lambda}\right)\right| \text {, for the case (5) } \\
C_{2}^{-}+\left|4 r_{0,1} r_{1,2} B \sin \left(2 \pi d_{2} n_{2} \cos \beta_{2} \frac{1}{\lambda}\right)\right|, \text { for the case (7) } \\
C_{1}^{+}+\left|4 r_{1,2} r_{2,3} B \cos \left(2 \pi d_{1} n_{1} \cos \beta_{1} \frac{1}{\lambda}\right)\right|, \text { for the case (9) } \\
C_{1}^{-}+\left|4 r_{1,2} r_{2,3} B \sin \left(2 \pi d_{1} n_{1} \cos \beta_{1} \frac{1}{\lambda}\right)\right|, \text { for the case (11) } \\
W^{\min }=\left\{\begin{array}{l}
C_{2}^{+}-\left|4 r_{0,1} r_{1,2} B \cos \left(2 \pi d_{2} n_{2} \cos \beta_{2} \frac{1}{\lambda}\right)\right|, \text { for the case (5) } \\
C_{2}^{-}-\left|4 r_{0,1} r_{1,2} B \sin \left(2 \pi d_{2} n_{2} \cos \beta_{2} \frac{1}{\lambda}\right)\right|, \text { for the case (7) } \\
C_{1}^{+}-\left|4 r_{1,2} r_{2,3} B \cos \left(2 \pi d_{1} n_{1} \cos \beta_{1} \frac{1}{\lambda}\right)\right|, \text { for the case (9) } \\
C_{1}^{-}-\left|4 r_{1,2} r_{2,3} B \sin \left(2 \pi d_{1} n_{1} \cos \beta_{1} \frac{1}{\lambda}\right)\right|, \text { for the case (11) }
\end{array}\right.
\end{array}\right.
$$

This fact may be easily proved in the same way as for the case of Eqs. (20) and (21), using the inequalities $W^{\max }-W \geq 0$ and $W-W^{\min } \geq 0$.

The analysis of the relations for $W^{\max , \min }$ shows that there are two possible functions for the envelopes of both the maximums and the minimums, which do not depend on the phase thickness of the thickest layer. The same conclusions are also true for the 
envelopes of the transmittance $\left(T^{\max , \min }=\frac{1}{W^{\min , \max }}\right)$ and the reflectance ( $R^{\max , \min }=1-\frac{1}{W^{\max , \min }}$ ) spectra. It is worth noticing that the regularity explained by us has in fact been used by the authors [9] when finding the functions of spectral envelopes for the structures with two layers. Later on it has been generalised for the multilayer structures (see $[10,15])$.

\subsection{Optical beatings in the reflectance and transmittance spectra of multilayer structures}

When passing to the analysis of multilayer structures, it is necessary to write down the expression for the peak-to-peak amplitude of the spectral oscillations, in order to define the conditions predetermining how clearly the optical beatings manifest themselves in the reflectance and transmittance spectra for an arbitrary multilayer structure. The following expressions for the envelopes of the reflectance and transmittance spectra have been obtained in the work [10]:

$$
\begin{gathered}
R_{s}^{\min , \max }=\left(\frac{\sigma_{0, s} \mp \sigma_{s, k+1} \chi_{0, s} \Omega_{s}}{1 \mp \sigma_{s, 0} \sigma_{s, k+1} \Omega_{s}}\right)^{2}, \\
T_{s}^{\min , \max }=\frac{n_{k+1}}{n_{0}} \frac{\cos \beta_{k+1}}{\cos \beta_{0}} \frac{\tau_{0, s}^{2} \tau_{s, k+1}^{2} \Omega_{s}}{\left(1 \pm \sigma_{s, 0} \sigma_{s, k+1} \Omega_{s}\right)^{2}},
\end{gathered}
$$

where $\Omega_{s}=e^{2 \operatorname{Im} \tilde{\delta}_{s}}, s$ denotes the number of the layer with the largest phase thickness, whereas $\chi_{j, u}, \sigma_{j, u}, \tau_{j, u}$ and $\gamma_{j, u}, \phi_{j, u}, \theta_{j, u}$ are respectively the modules and the phases of the complex quantities $\tilde{t}_{j, u} \tilde{t}_{u, j}-\tilde{r}_{j, u} \tilde{r}_{u, j}=\chi_{j, u} e^{i \gamma_{j, u}}, \quad \tilde{r}_{j, u}=\sigma_{j, u} e^{i \phi_{j, u}}$ and $\tilde{t}_{j, u}=\tau_{j, u} e^{i \theta_{j, u}}$. Here $\tilde{r}_{j, u}$ and $\tilde{t}_{j, u}$ are the reflection and transmission coefficients for the structure $j-(j+1)-\cdots-u$, and $n_{0}$ and $n_{k+1}$ mean the refractive indices of transparent semi-infinite surrounding media. As a consequence, the relations

$$
\begin{aligned}
& \Delta R_{s}=R_{s}^{\max }-R_{s}^{\min }=\frac{4 \Omega_{s} \sigma_{s, k+1}\left(\sigma_{0, s} \sigma_{s, 0}-\chi_{0, s}\right)\left(\sigma_{s, 0} \sigma_{s, k+1}^{2} \chi_{0, s} \Omega_{s}^{2}-\sigma_{0, s}\right)}{\left(\sigma_{s, 0} \sigma_{s, k+1} \Omega_{s}-1\right)^{2}\left(\sigma_{s, 0} \sigma_{s, k+1} \Omega_{s}+1\right)^{2}}, \\
& \Delta T_{s}=T_{s}^{\max }-T_{s}^{\min }=\frac{n_{k+1}}{n_{0}} \frac{\cos \beta_{k+1}}{\cos \beta_{0}} \frac{4 \Omega_{s}^{2} \sigma_{s, 0} \sigma_{s, k+1} \tau_{0, s}^{2} \tau_{s, k+1}^{2}}{\left(\sigma_{s, 0} \sigma_{s, k+1} \Omega_{s}-1\right)^{2}\left(\sigma_{s, 0} \sigma_{s, k+1} \Omega_{s}+1\right)^{2}}
\end{aligned}
$$

may be written for the peak-to-peak amplitudes between the envelopes given by Eqs. (22) and (23). 
The analysis of the latter expressions testifies that the minimum values of the ratios $\frac{\Delta R_{s}^{\min }}{\Delta R_{s}^{\max }}$ (i.e., $\Delta R_{s} \rightarrow 0$ for the reflectance spectrum) are reached when one of the following conditions is satisfied:

(1) The module of the reflection coefficient for the structure $s-(s+1)-\cdots-(k+1)$ is close to zero $\left(\sigma_{s, k+1} \rightarrow 0\right)$;

(2) $\sigma_{s, 0} \sigma_{s, k+1}^{2} \chi_{0, s} \Omega_{s}^{2}-\sigma_{0, s} \rightarrow 0$. The relations $\tilde{r}_{j, u}=-\tilde{r}_{u, j}^{*} \exp \left(i \gamma_{j, u}\right) \quad$ and $\tilde{t}_{j, u} \tilde{t}_{u, j}-\tilde{r}_{j, u} \tilde{r}_{u, j}=\exp \left(i \gamma_{j, u}\right)$ are true for the transparent structure [16]. Then we have $\sigma_{s, 0}=\sigma_{0, s}$ and $\chi_{0, s}=1$, and the condition $\sigma_{s, 0} \sigma_{s, k+1}^{2} \chi_{0, s} \Omega_{s}^{2}-\sigma_{0, s} \rightarrow 0$ may be rewritten as $\sigma_{0, s}\left(\sigma_{s, k+1}^{2}-1\right) \rightarrow 0$. This is satisfied at either $\sigma_{0, s} \rightarrow 0$ or $\sigma_{s, k+1} \rightarrow 1$.

(3) $\sigma_{0, s} \sigma_{s, 0}-\chi_{0, s} \rightarrow 0$. For the transparent structure this condition is equivalent to the condition $\sigma_{0, s} \rightarrow 1$.

The minimum values of the ratios $\frac{\Delta T_{s}^{\min }}{\Delta T_{s}^{\max }}$ (i.e., $\Delta T_{s} \rightarrow 0$ for the transmittance spectrum) are reached when one of the following conditions is fulfilled:

(1) $\sigma_{s, k+1} \rightarrow 0$; (2) $\sigma_{s, 0} \rightarrow 0$; (3) $\tau_{0, s} \rightarrow 0$; (4) $\tau_{s, k+1} \rightarrow 0$.

The minimums $\frac{\Delta R_{s}^{\min }}{\Delta R_{s}^{\max }}$ of the reflectance spectrum and the minimums $\frac{\Delta T_{s}^{\min }}{\Delta T_{s}^{\max }}$ of the transmittance spectrum for the transparent structure are reached when we have the conditions of zero reflectance for one of the two parts of the structure $0-1-\cdots-(k+1)$ separated by the thickest layer $s: 0-1-\cdots-s$ or $s-(s+1)-\cdots-(k+1)$ (see Fig. 4). In case if one of these parts represents a single-layer structure ( $s=2$ or $s=k-1$ ), the optical beating manifests itself very clearly when the known antireflection conditions are satisfied for such a structure [17]:

$$
N_{1}=\sqrt{N_{0} N_{2}}, \delta_{1}=\pi / 2+\pi \zeta
$$

or

$\zeta=0,1, \ldots$ for $s=2$

$$
N_{0}=N_{2}, \delta_{1}=\pi \zeta
$$

or

$$
N_{k}=\sqrt{N_{k-1} N_{k+1}}, \delta_{k}=\pi / 2+\pi \zeta
$$

for $s=k-1$ ).

$$
N_{k-1}=N_{k+1}, \delta_{k}=\pi \zeta,
$$

Here $N_{i}$ are the generalised refractive indices defined as $N_{i}=n_{i} \cos \beta_{i}$ for the $s$ polarisation and $N_{i}=\frac{\cos \beta_{i}}{n_{i}}$ (for $p$-polarization) [11] 
It presents no difficulty to make sure that the conditions for the generalised refractive indices given by Eqs. (28)-(31) correspond to the conditions (9), (11), (5), (7) for the Fresnel reflection coefficients.

In case if one of the mentioned parts represents a multilayer structure, the optical beating appears to be clear (see Fig. 4) when the phase thicknesses of any of the two layers in this part satisfy the conditions of zero reflectance [10, 18].

The optical beating is also clearly present when the transmittances of the structures $0-1-\cdots-s$ or $s-(s+1)-\cdots-(k+1)$ are minimal (see Fig. 5), while their reflectances are close to unity (i.e., we have an 'interference reflector').

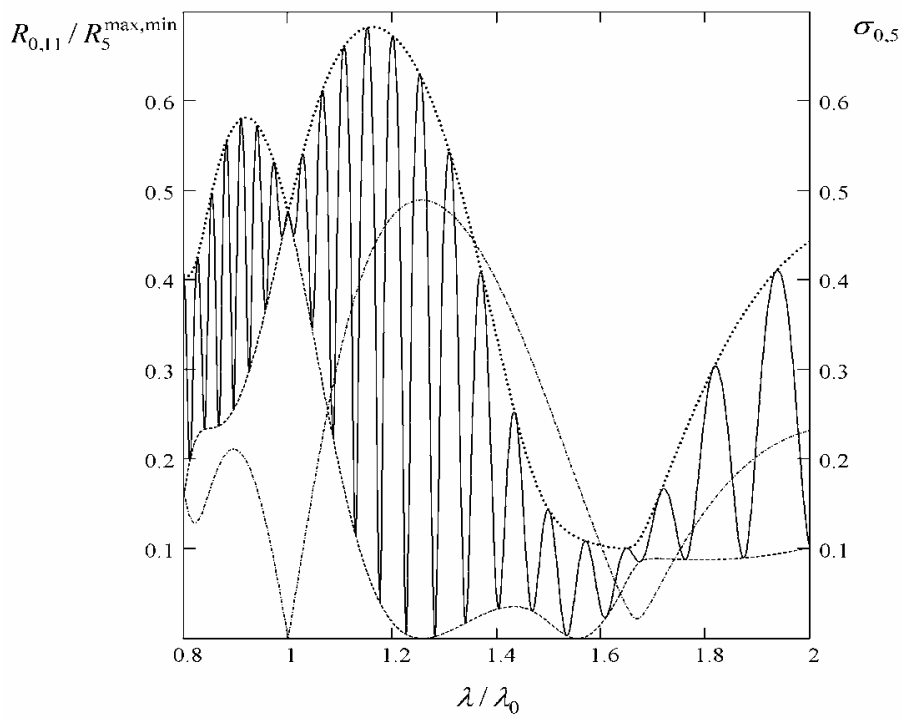

Fig. 4. Reflectance at normal incidence ( $R_{0,11}$, solid curve) and envelopes of its minimums ( $R_{5}^{\min }$, dashed curve) and maximums ( $R_{5}^{\max }$, dotted curve) for a transparent ten-layer structure with the thickest fifth layer and the following parameters at $\lambda=\lambda_{0}: n_{0}=1, n_{1}=1.37, n_{2}=1.70, n_{3}=1.98, n_{4}=2.95$, $n_{5}=1.97, \quad n_{6}=2.90, \quad n_{7}=1.90, \quad n_{8}=2.20, \quad n_{9}=1.50, \quad n_{10}=2.70$, $n_{11}=4.0 ; \delta_{1}=0.927 \mathrm{rad}, \delta_{2}=1.697 \mathrm{rad}, \delta_{3}=1.640 \mathrm{rad}, \delta_{4}=5.854 \mathrm{rad}$, $\delta_{5}=81.52 \mathrm{rad}, \quad \delta_{6}=1.571 \mathrm{rad}, \quad \delta_{7}=1.571 \mathrm{rad}, \quad \delta_{8}=1.351 \mathrm{rad}$, $\delta_{9}=1.293 \mathrm{rad}, \delta_{10}=1.236 \mathrm{rad}$. A zero $\sigma_{0,5}$ value is provided at $\lambda=\lambda_{0}$ (dash-dotted curve).

\section{Conclusions}

A possible occurrence of an optical beating in the inverse transmittance spectrum for a transparent double-layer structure has been demonstrated using an analogy between analytical representations of the latter spectrum and the beating phenomenon known from the standard vibration theory. Once being drawn, the analogy has enabled us deriving analytical expressions for the envelopes of both the maximums and the minimums in the inverse transmittance spectrum. There are two different expressions for the envelopes that 
correspond to the two possible ratios between the phase thicknesses of the layers, $\delta_{1}>\delta_{2}$ and $\delta_{1}<\delta_{2}$.

The analytical conditions needed for clear manifestations of the optical beatings both in the reflectance $\left(\Delta R_{s} \rightarrow 0\right)$ and transmittance $\left(\Delta T_{s} \rightarrow 0\right)$ spectra of an arbitrary multilayer structure have been determined. For the transparent structures these conditions mean a closeness to zero or unity of the reflectance of any part of the structure $0-1-\cdots-(k+1)$ separated by the thickest layer $s \quad(0-1-\cdots-s$ or $s-(s+1)-\cdots-(k+1))$.

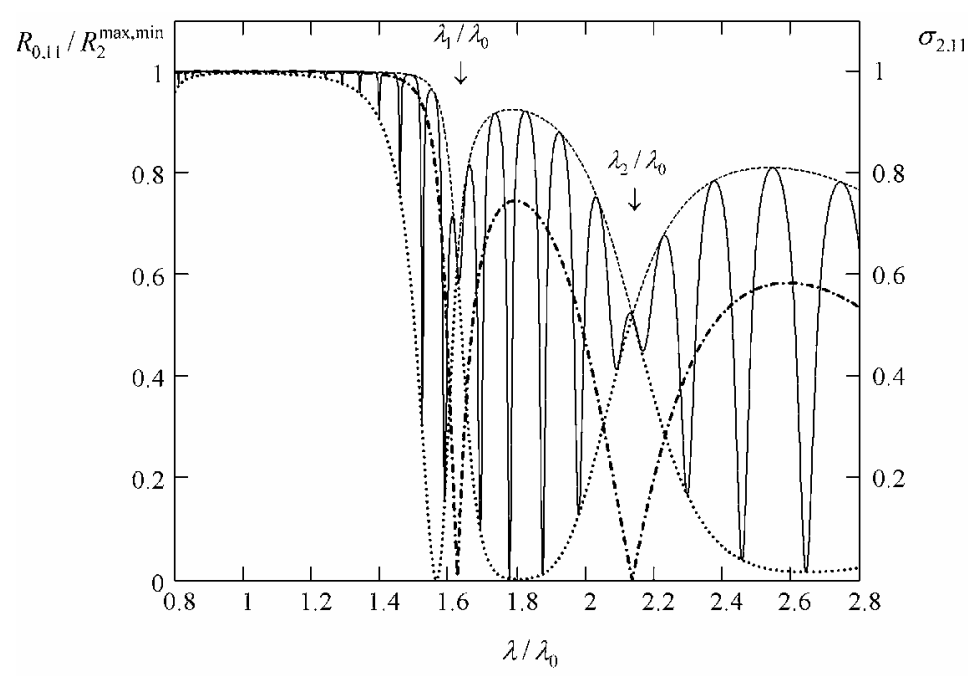

Fig. 5. Reflectance at normal incidence ( $R_{0,11}$, solid curve) and envelopes of its minimums ( $R_{2}^{\min }$, dashed curve) and maximums ( $R_{2}^{\max }$, dotted curve) for a transparent ten-layer structure with the thickest second layer and the following parameters at $\lambda=\lambda_{0}: n_{0}=1, \quad n_{1}=n_{3}=n_{5}=n_{7}=n_{9}=n_{11}=4.0$, $n_{2}=n_{4}=n_{6}=n_{8}=n_{10}=1.40 ; \delta_{1}=\delta_{3}=\delta_{4}=\cdots=\delta_{10}=\pi / 2, \delta_{2}=101.5 \mathrm{rad}$. A value $\sigma_{2,11} \approx 1$ and a zero $\sigma_{2,11}$ value (dash-dotted curve) are provided at $\lambda=\lambda_{0}$ and $\lambda=\left(\lambda_{1}, \lambda_{2}\right)$, respectively.

\section{References}

1. Tikhonravov A V and Trubetskov M K, 2007. Modern status and prospects of the development of methods of designing multilayer optical coatings. J. Opt. Technol. 74: 845-850.

2. Alboon S A and Lindquist R G, 2008. Flat-top liquid crystal tunable filter using coupled Fabry-Perot cavities. Opt. Express. 16: 231-236.

3. Li J, Abolghasemi L E, Herman P R, Kitaev V and Ozin G A, Colloidal photonic crystal mirrors for high resolving-power Fabry-Perots. OSA Conf. on Lasers and Electronics (CLEO), San Francisco, CA, 16-24 May 2004. P.CtuDD2. 
4. Yalcin A, Popat K C, Aldridge J C, Desai T A, Hryniewicz J, Chbouki N, Little B E, King Oliver, Van V, Chu Sai, Gill D, Anthes-Washburn M, Unlu M S and Goldberg B B, 2006. Optical sensing of biomolecules using microring resonators. IEEE J. Selected Topics Quantum Electron. 12: 148-155.

5. J Yi, 2008. High-resolution interrogation technique for fiber optic extrinsic FabryPerot interferometric sensors by the peak-to-peak method. Appl. Opt. 47: 925-932.

6. Macleod H A. Thin-film optical filters. Bristol and Philadelphia: Institute of Physics Publishing, Chapter 9 (2003).

7. F Lai, Y Wang, M Li, H Wang, Y Song and Y Jiang, 2007. Determination of optical constants and inhomogeneity of optical films by two-step film envelope method. Thin Solid Films. 515: 4763-4767.

8. Martinez-Anton J C and Esteban O, 2004. Photo-interferometric spectroscopic ellipsometry. Thin Solid Films. 455-456: 90-94.

9. Kosobutskyy P S and Kushnir O P, 2008. Envelopes of optical interference spectra for duplex and triplex layered structures. Ukr. J. Phys. Opt. 9: 73-81.

10. Kushnir O P, 2009. Application of spectral envelope functions of multilayer structures for analytical determination of antireflection conditions. Ukr. J. Phys. Opt. 10: $82-88$.

11. Kard P, Analysis and synthesis of multilayer interference coatings. Tallinn: Valgus (1971).

12. Savelyev I V, General course of physics, Vol. 1 (Mechanics, vibrations and waves, and molecular physics). Moscow: Nauka (1970).

13. Ios G, Course of theoretical physics, Part 1 (Mechanics and electrodynamics). Moscow: Uchpedgiz (1963).

14. Born M and Wolf E, Principles of optics. Moscow: Nauka (1970).

15. Kosobutskyy P S, 2008. Analytical functions of the envelopes of Fabry-Perot spectra of the multilayer structures. Pisma Zhurn. Tekhn. Fiziki. 34: 79-82.

16. Monz'on J J and S'anchez-Soto L L, 1999. Lossless multilayers and Lorentz transformations: more than an analogy. Opt. Commun. 162: 1-6.

17. Grebenshchikov I V, Vlasov A G, Neporent B S and Sujkovsky N V, Non-reflective coatings on optical materials. Moscow: OGIZ (1946).

18. Kushnir O P, 2010. Conditions for the extremal reflection of multilayer structures: application for designing of narrow bandpass filters. J. Phys. Stud. 14: 4401-4411.

Kosobutskyy P.S., Bilyj J.M. and Kushnir O.P., 2011. Demonstration of optical beating in the reflectance and transmittance spectra of multilayer structures Ukr.J.Phys.Opt. 12: 89-100.

Анотація. Представлено аналогію між оптичним биттям в обернених спектрах пропускання прозорої двошарової структури і явищем биття, відомим з теорії коливань. Визначено умови, за яких оптичне биття найчіткіше виявляється в інтерференційних спектрах для багатошарової структури. 